\title{
A Realistic Anthropomorphic Dynamic Heart Phantom
}

\author{
R Haddad, P Clarysse, M Orkisz, P Croisille, \\ D Revel, IE Magnin

\section{Creatis, CNRS UMR \#5515, INSERM U630, F-69621 Villeurbanne Cedex, France}

\begin{abstract}
This paper presents a realistic numerical beating heart phantom. The development of such a model is motivated by the need of an appropriate ground truth reference for the evaluation of cardiac image processing algorithms. An anatomical model has been developed by extracting cardio-vascular structures contours from a $3 D$ acquisition in Magnetic Resonance Imaging (MRI) of a human healthy subject. Cardiac motion has been tracked by $3 D$ non rigid registration between the consecutive linearly interpolated volumes from dynamic MRI sequences. The so-obtained numerical phantom is structurally rich and its motion realistic. As it issues from the same MRI acquisition on the same human healthy subject, it presents a very good overall consistency and will be use for cardiac image processing algorithms evaluation.
\end{abstract}

\section{Introduction}

The recent advances in cardiac imaging modalities give access to the heart's anatomy and function with an increasing accuracy in 3D and through the cardiac cycle. This allows studying ischemic diseases, which are the most common cause of death in developed countries. For diagnostic purposes, clinicians need image postprocessing techniques in order to obtain quantitative functional parameters, currently cavity volumes, ejection fraction or local motion indexes such as myocardial wall thickening. Therefore, several methods have been developed to estimate the heart shape and motion [1-3]. However, the performance comparison of theses methods is very difficult to achieve as usually in medical image analysis no ground truth exists. In this paper we propose a numerical dynamic model of the heart that can serve as a reference to objectively evaluate cardiac segmentation and motion estimation methods Error! Reference source not found. Such a reference model can also be of a great interest for the evaluation of image reconstruction [4] and registration Error! Reference source not found. algorithms. In these cases, the model includes a computer description of the cardiac structures and motion as well as the associated multimodality medical images. Simulation of minimally invasive beating heart surgeries could also benefit from this model [6].

\section{Methods}

The development of the numerical phantom basically relies on two steps. First, a structurally rich anatomical static heart model is build from a 3D anatomical MR acquisition on a healthy volunteer. Then 3D heart motion is estimated from MRI dynamic series on the same subject and applied to the anatomical model. The process is summed up in Fig. 1.

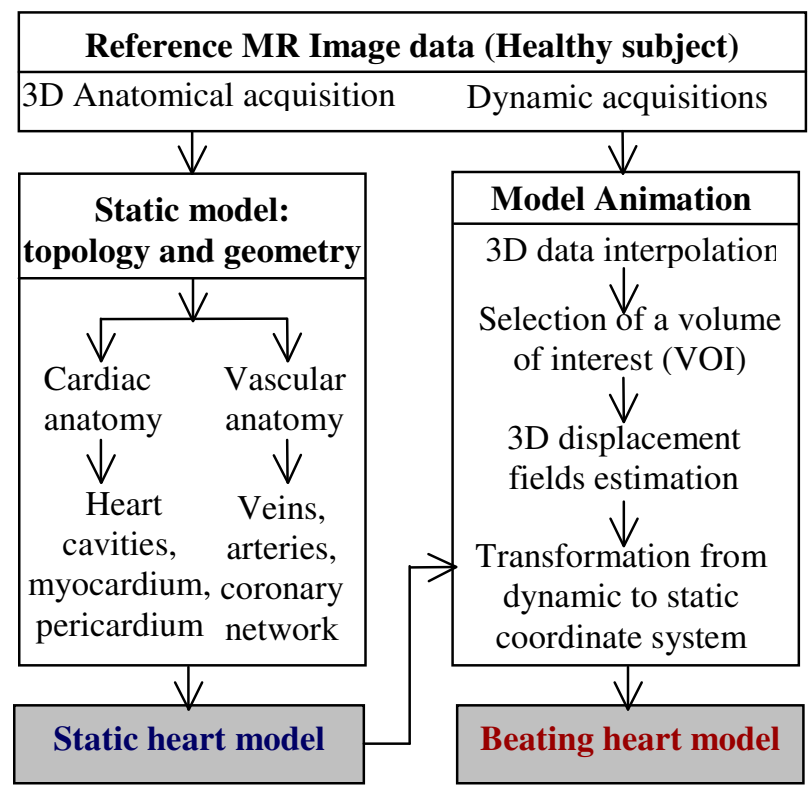

Fig. 1. Overview of the anthropomorphic beating heart model development steps

\subsection{Data sets}

For the static model building, a 3D MRI acquisition has been performed on a healthy subject (Fig. 2 (a)). 3D sense sequence has been used with ECG gating and echo 
navigation (free respiration) to acquire a dense set of 80 transverse slices (slice thickness $=2 \mathrm{~mm}$, spacing between slices $=1 \mathrm{~mm}$, image resolution $=0.53 \times 0.53 \mathrm{~mm}^{2}$, matrix $=512 \times 512)$ to cover the whole heart and peripheral vessels at mid-systole. During the same examination, cine images have been acquired on the same volunteer, to be used for the animation of the model. Seven short axis slices cover the whole heart. For each slice, thirty frames sample the cardiac cycle. A total of 210 short axis images have thus been obtained with the following parameters: slice thickness $=7 \mathrm{~mm}$, slice spacing $=10 \mathrm{~mm}$, slice resolution $=1.05 \times 1.05 \mathrm{~mm}^{2}$, matrix $=256 \times 256($ Fig. $2(\mathrm{~b})$ (c)).
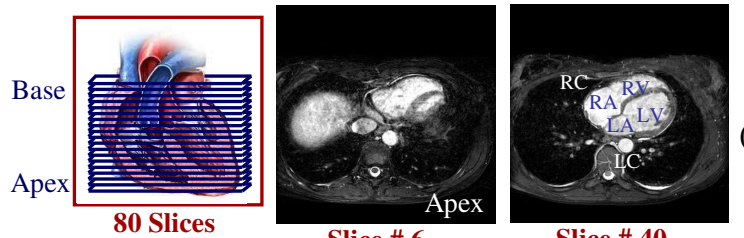

(a) Slice \# 6 Slice \# 40
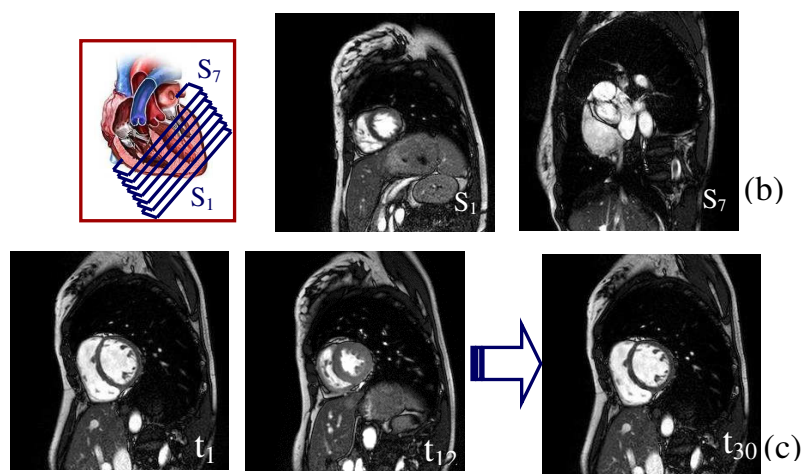

Fig. 2. Reference MRI data: (a) 2 slices of the dense 3D anatomical acquisition. (b) 2 levels of short axis slices. (c) 3 over the 30 frames acquired during the cardiac cycle at one short axis slice level.

\subsection{Static model construction}

The contours of the different cardiac structures, left and right ventricles and atria (LV, RV, LA, RA), the pericardium, have been manually traced by a radiologist. The contours have been individually checked and labelled. In the same way, the junctions of the four pulmonary veins, vena cava and the aorta have been segmented. Some of the extracted contours are shown in Fig.3 (a). Next, 3D cardiac structures reconstruction is realised by the Nuages software (http://wwwsop.inria.fr/prisme/). Surfaces are available as 3D triangle meshes. A smoothed version of the surfaces has been generated using Laplacian smoothing. Fig.3 (b) shows some of the reconstructed surfaces. Semi-automatic extraction and reconstruction of the coronary network in the data set was achieved in the following steps: 1) image filtering aiming at the enhancement of the coronary arteries, while attenuating other structures, 2) segmentby-segment extraction of arterial centrelines, along with estimation of local diameters, 3) extraction of crosssectional lumen boundaries of each arterial segment in image planes locally perpendicular to the centreline, 4) connection and smoothing of the centreline segments, 5) generation of smoothed lumen surfaces. This method is implanted in the MARACAS software developed in our laboratory (Fig.3 (c)) [7].

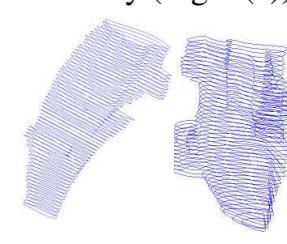

(a)

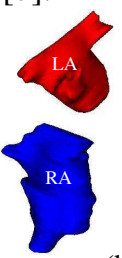

(b

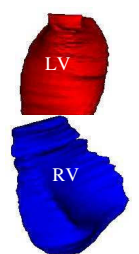

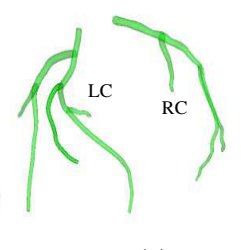

(c)
Fig. 3. Static model construction: (a) Extracted 3D contours, (b) Reconstructed 3D cardiac surfaces, (c) Reconstructed coronary network.

The different cardiac and vascular anatomical structures have been finally combined. Fig. 4 illustrates the obtained $3 \mathrm{D}$ anatomical model and shows the correspond-dence with anatomical sheets.
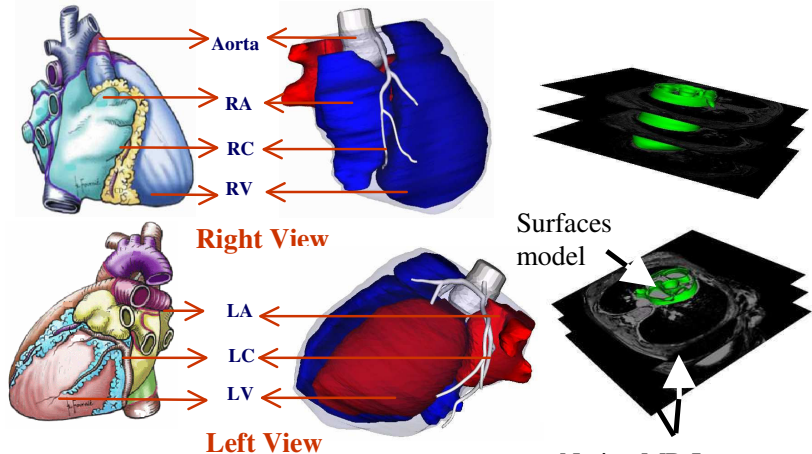

Native MR Images

(a)

(b)

Fig. 4. (a) 3D static heart model in relation with anatomical sheets. (b) Views of the correspondence between transverse reference $\mathrm{MR}$ images and the 3D static model.

\subsection{Cardiac motion estimation}

Cardiac motion has been estimated by non rigid image registration between MR frames in the sequence (30 frames) acquired on the same volunteer as for the static model (Fig.2 (b), (c)). The method allows recovering 
global as well as local motion features which characterize the heart dynamics. First, images at each time point are linearly interpolated to an isotropic 3D volume. A volume of interest (VOI) has been selected to focus on the heart area and to reduce the computing time. Consecutive volumes pairs are then matched using a 3D non rigid registration method based on volumic free form deformations (3D-FFD). Image transformation with FFD is iterated through the displacement of grid control points until the minimum of an intensity similarity criterion between the floating image $F$ and a reference image $R$ is reached. Let $\phi$ be a regular grid of control points of size $\mathrm{n}_{\mathrm{x}} \times \mathrm{n}_{\mathrm{y}} \times \mathrm{n}_{\mathrm{z}}$. The 3D FFD transformation is defined by:

$$
T_{F F D}(x, y, z)=\sum_{l=0}^{3} \sum_{m=0}^{3} \sum_{n=0}^{3} B_{l}(u) B_{m}(v) B_{n}(w) \phi_{i+l, j+m, k+n}
$$

where $B_{l}$ represents the $l_{t h}$ basis function of the B-spline,

$$
\text { and } \begin{aligned}
i & =\left\lfloor x / n_{x}\right\rfloor-1, j=\left\lfloor y / n_{y}\right\rfloor-1, k=\left\lfloor z / n_{z}\right\rfloor-1 \\
u & =x / n_{x}-\left\lfloor x / n_{x}\right\rfloor, v=y / n_{y}-\left\lfloor y / n_{y}\right\rfloor, \mathrm{w}=z / n_{z}-\left\lfloor z / n_{z}\right\rfloor,
\end{aligned}
$$

with \lfloor\rfloor the floor operator Error! Reference source not found..

As the matched images are of the same nature, we chose the sum of squared intensity differences (SSD) as the registration metric:

$$
S S D=\frac{1}{N} \sum_{i \in \Omega_{A, B}^{T}}|R(i)-F(i)|^{2}
$$

Where the intensities in the reference and transformed floating images are $R(i)$ and $F(i)$,respectively, and $N$ is equal to the number of pairs of pixels in the intersection region between the two images. The minimum of this criterion is searched for using a gradient descent technique. The algorithm stops when the similarity stabilizes or when a maximum iteration number is reached. In practice, the parameters were fixed to the following values: the grid size is $20 \times 20 \times 20$ pixels, the maximum iteration number is 30 , the maximum step length of the optimizer is 15 and VOI dimension is $128 \times 128 \times 70$ voxels. We designed the algorithm from software components of the ITK (http://www.itk.org) library. Fig. 5 illustrates the deformed grid at two instants of the cardiac cycle.

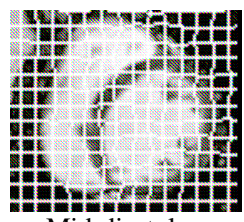

Mid-diastole

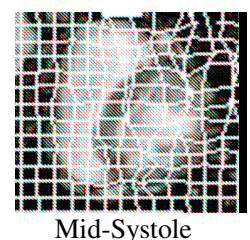

Mid-Systole
Fig. 5. FFD deformed grid overlaid onto the floating image (2D slice of a $3 \mathrm{D}$ volume).

Fig. 6 shows displacement fields that have been computed at three instants of the cardiac cycle

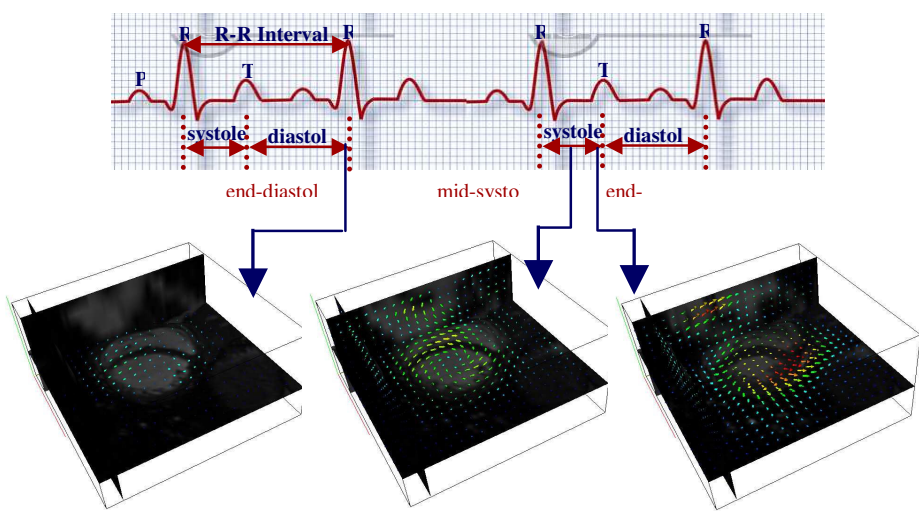

Fig. 6. 2D displays of computed 3D displacement fields from non rigid image registration

\subsection{Model animation}

Non Rigid registration is iterated for each successive VOI pairs in the sequence. This results in a set of 30 steps of $3 \mathrm{D}$ control point displacements. The model animation consists in applying the retrieved FFDs to the static model. To this end, the model is transformed to the cine short axis image coordinate system, using image position and orientation available in image file headers. This assumes that the subject has not moved between the two acquisitions which have been visually checked in our image data set. Then, the successive FFD warpings are applied to the reoriented static model to simulate the heart motion. Each deformed model configuration is recorded in a separate file. Fig. 7 illustrates three instants of the pericardium dynamics resulting from the application of the estimated movement onto the pericardium at the reference time frame 0 .

\section{Discussion and conclusions}

A structurally rich $3 \mathrm{D}$ realistic beating model of the heart and main vessels has been presented in this paper. A few heart models have been previously reported in the literature. Some of them issue from the biomechanical community [9] but the geometry of the heart structures are quite idealized and usually restricted to the LV. The NCAT model [9] and the model presented in [6] are similar to the one proposed in this paper. The main difference relies on the fact that, in our model, all the imaging data, including heart and vessels anatomy and 
heart's motion, have been recorded from the same human patient, with the same MRI modality and during the same examination. This insures a very good consistency of the resulting model. Moreover, the model includes the native
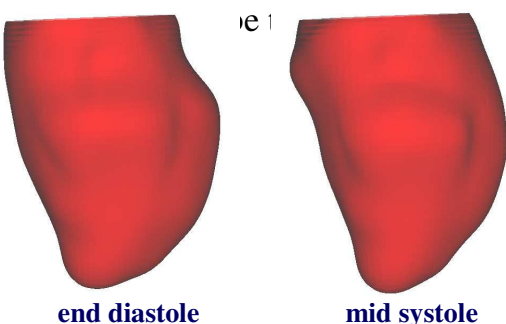

(a)

Pericardium volume

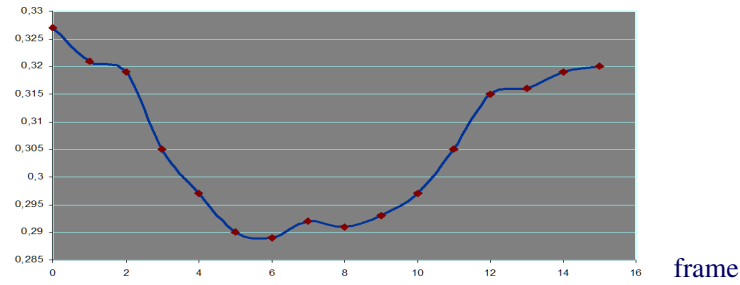

(b)

Fig. 7. (a) Pericardium at 3 instants of the cardiac cycle (b) volume evolution over the cardiac cycle

motion model. Therefore, the overall model composed of the structure surfaces, the images and the motion model, constitutes a very good numerical reference phantom to validate cardiac image processing algorithms. Still, the present model has to be improved and completed. The model itself should be validated by evaluating the segmented contours and 3D estimated motion. Also, this phantom will be virtually imaged by using modality simulators (Positron Emission Tomography, MRI...) Error! Reference source not found., [10]. Simulating various heart dynamics can be achieved either by adequately tuning the model's parameters (control points) or processing additional examinations for healthy volunteers and pathological subjects. We then truly hope to deliver these realistic models to the community for evaluation of cardiac image processing algorithms, especially 4D cardiac segmentation methods Error! Reference source not found.,[11].

\section{References}

[1] Pham Q.-C., Vincent F., Clarysse P., Croisille P., Magnin I.E. A FEM based deformable model for the 3D segmentation and tracking of the heart in cardiac MRI. In: 2nd International Symposium on Image and Signal Processing and Analysis; 2001; Pula, Croatia; 2001. p. 250-
254.

[2] Frangi A.F., Niessen W.J., Viergever M.A. Threedimensional modelling for functional analysis of cardiac images: a review. IEEE Transactions on Medical Imaging 2001;20:2-25.

[3] Jasjit S. Suri. Computer vision, pattern recognition and image processing in left ventricle segmentation: the last 50 years. Pattern Analysis and Applications 2000;3:209-242.

[4] Chalana V., Yongmin K. A methodology for evaluation of boundary detection algorithms on medical images. IEEE Transactions on Medical Imaging 1997;16(5):642-652.

[5] Grangeat P., Koenig A., Rodetand T., Bonnet S. Theoretical framework for a dynamic cone-beam reconstruction algorithm based on a dynamic particle model. Physics in Medicine and Biology 2002;47:26112625.

[6] Pauna N., Croisille P., Costes N., Reilhac A., Mäkelä T., Cozar O., et al. A strategy to quantitatively evaluate MRI/PET cardiac rigid registration methods using a monte carlo simulator. In: Functional Imaging and Modeling of the Heart; 2003: Springer, Lecture Notes in Computer Science; 2003. p. 194-204.

[7] Wierzbicki M., Drangova M., Guiraudon G., Peters T. Validation of dynamic heart models obtained using nonlinear registration for virtual reality training, planning, and guidance of minimally invasive cardiac surgeries. Medical Image Analysis 2004;8:387-401.

[8] Hernàndez-Hoyos M., Orlowski P., Piatkowska-Janko E., Bogorodzkiand P., Orkisz M. Vascular centreline extraction in 3D MR angiograms to optimise acquisition plane for blood flow measurement by phase contrast MRI. In: Computer Assisted Radiology and Surgery; 2005; Berlin (Germany); 2005. p. 345-350.

[9] Rueckert D., Sonoda L.I., Hayes C., Hill D.L.G., Leach M.O., Hawkes D.J. Nonrigid registration using free-form deformations: Application to breast MR images. IEEE Transactions on Medical Imaging 1999;18(8):712-721.

[10] Segars W.-P., Lalush D.-S., Tsui B.-M.-W. A realistic spline-based dynamic heart phantom. In: IEEE Nuclear Science Symposium and Medical Imaging Conference; 1999; 1999. p. 503-506.

[11] Reilhac A., Lartizien C., Costes N., Comtat C., Evans A.C PET-SORTEO: A Monte Carlo-based simulator with high count rate capabilities. IEEE Transactions on Nuclear Science 2004;51(1):46-52.

[12] Montagnat J., Delingette H. 4D deformable models with temporal constraints: application to 4D cardiac image segmentation. Medical Image Analysis 2005;9(1):87-100.

Address for correspondence

Rana HADDAD

CREATIS, CNRS UMR \#5515, INSERM U630

INSA de Lyon

Bâtiment Blaise Pascal

7, Avenue Jean Capelle

69621 Villeurbanne Cedex (France)

rana.haddad@creatis.insa-lyon.fr 